DOI: $10.15393 / \mathrm{j} 3$.art.2021.8530

UDC 517.58, 517.52, 512.62

H. Merzouk, B. Aloui, A. Boussayoud

\title{
GENERATING FUNCTIONS OF THE PRODUCT OF 2-ORTHOGONAL CHEBYSHEV POLYNOMIALS WITH SOME NUMBERS AND THE OTHER CHEBYSHEV POLYNOMIALS
}

\begin{abstract}
In this paper, we give the generating functions of binary product between 2-orthogonal Chebyshev polynomials and $k$ Fibonacci, $k$-Pell, $k$-Jacobsthal numbers and the other orthogonal Chebyshev polynomials.

Key words: 2-Orthogonal Chebyshev polynomials; $k$-Fibonacci; $k$-Pell and $k$-Jacobsthal numbers; Generating functions; Chebyshev polynomials
\end{abstract}

2010 Mathematical Subject Classification: 33C45, 42C05

1. Introduction. The Chebyshev polynomials $T_{n}(x), U_{n}(x), V_{n}(x)$ and $W_{n}(x), n \geqslant 0$, of the first, second, third and fourth kinds are respectively defined by the following formulas:

$$
\begin{gathered}
T_{n}(\cos \theta)=\cos (n \theta), \quad U_{n}(\cos \theta)=\frac{\sin [(n+1) \theta]}{\sin \theta}, \\
V_{n}(\cos \theta)=\frac{\cos (n+1 / 2) \theta}{\cos (\theta / 2)}, \quad W_{n}(\cos \theta)=\frac{\sin (n+1 / 2) \theta}{\sin (\theta / 2)},
\end{gathered}
$$

where $x=\cos \theta, \theta \in[0, \pi]$. (For more details see [9], [10]).

The resulting polynomials, $T_{n}(x), U_{n}(x), V_{n}(x)$ and $W_{n}(x)$ are multiples of the Jacobi polynomials. In fact, $T_{n}(x)=P_{n}^{\left(-\frac{1}{2},-\frac{1}{2}\right)}(x), U_{n}(x)=$ $=P_{n}^{\left(\frac{1}{2}, \frac{1}{2}\right)}(x), V_{n}(x)=P_{n}^{\left(-\frac{1}{2}, \frac{1}{2}\right)}(x), W_{n}(x)=P_{n}^{\left(\frac{1}{2},-\frac{1}{2}\right)}(x), n \geqslant 0$, where $\left\{P_{n}^{(\alpha, \beta)}\right\}_{n \geqslant 0},(\alpha, \beta \neq-m, \alpha+\beta \neq-m-1, m \geqslant 1)$, is the Jacobi polynomials given by the following explicit expression [7], [15]

$$
P_{n}^{(\alpha, \beta)}(x)=\sum_{\nu=0}^{n}\left(\begin{array}{l}
n \\
\nu
\end{array}\right) \frac{2^{n-\nu} \Gamma(n+\alpha+\beta+\nu+1) \Gamma(n+\beta+1)}{\Gamma(2 n+\alpha+\beta+1) \Gamma(\nu+\beta+1)}(x-1)^{\nu} .
$$

(c) Petrozavodsk State University, 2021 
There is the following simple relations between the Chebyshev polynomials

$$
\begin{aligned}
T_{n+1}^{\prime}(x) & =(n+1) U_{n}(x), n \geqslant 0 . \\
V_{n}(x) & =U_{n}(x)-U_{n-1}(x), n \geqslant 0 . \\
W_{n}(x) & =U_{n}(x)+U_{n-1}(x), n \geqslant 0 .
\end{aligned}
$$

It is well known that Chebyshev polynomials are orthogonal, symmetric and satisfy the following Three-Term Recurrence Relation (TTRR)

$$
P_{n+2}(x)=x P_{n+1}(x)-\gamma_{n+1} P_{n}(x), n \geqslant 0,
$$

with initial conditions $P_{0}(x)=1, P_{1}(x)=x$.

In the following table, we give for $n \geqslant 0$, the explicit expression of the parameters involved in (1) of the monic Chebyshev polynomials (for more details, see [11], [12]).

\begin{tabular}{|c|c|c|}
\hline$P_{n}(x)$ & TTRR & Initial conditions \\
\hline$T_{n}(x)$ & $T_{n+2}(x)=x T_{n+1}(x)-\frac{2^{\delta_{n}, 0}}{4} T_{n}(x)$ & $T_{0}(x)=1, T_{1}(x)=x$ \\
\hline$U_{n}(x)$ & $U_{n+2}(x)=x U_{n+1}(x)-\frac{1}{4} U_{n}(x)$ & $U_{0}(x)=1, U_{1}(x)=x$ \\
\hline$V_{n}(x)$ & $V_{n+2}(x)=x V_{n+1}(x)-\frac{2^{\delta_{n}, 0}}{4} V_{n}(x)$ & $V_{0}(x)=1, V_{1}(x)=x$ \\
\hline$W_{n}(x)$ & $W_{n+2}(x)=x W_{n+1}(x)-\frac{1}{4} W_{n}(x)$ & $W_{0}(x)=1, W_{1}(x)=x$ \\
\hline
\end{tabular}

Table 1: Some characteristic of Chebyshev polynomials.

It can be easily seen from the (1) that the generating functions for $T_{n}(x), U_{n}(x), V_{n}(x)$ and $W_{n}(x)$ are respectively given by (see [9], [10], [13])

$$
\begin{array}{ll}
\frac{1-x t}{1-2 x t+t^{2}}=\sum_{n=0}^{\infty} T_{n}(x) t^{n}, & \frac{1}{1-2 x t+t^{2}}=\sum_{n=0}^{\infty} U_{n}(x) t^{n}, \\
\frac{1-t}{1-2 x t+t^{2}}=\sum_{n=0}^{\infty} V_{n}(x) t^{n}, & \frac{1+t}{1-2 x t+t^{2}}=\sum_{n=0}^{\infty} W_{n}(x) t^{n} .
\end{array}
$$

Now, we recall the notion of $d$-orthogonal polynomials. A remarkable characterization of the $d$-monic orthogonal polynomial sequence is that 
those sequences satisfy a $(d+1)$-order recurrence relation, which we write in the form

$$
P_{m+d+1}(x)=\left(x-\beta_{m+d}\right) P_{m+d}(x)-\sum_{\nu=0}^{d-1} \gamma_{m+d-\nu}^{d-1-\nu} P_{m+d-1-\nu}(x), m \geqslant 0,
$$

with the initial conditions $P_{0}(x)=1, P_{-1}(x)=0$ and if $d \geqslant 2$

$$
P_{n}(x)=\left(x-\beta_{n-1}\right) P_{n-1}(x)-\sum_{\nu=0}^{n-2} \gamma_{d-1-\nu}^{n-1-\nu} P_{n-2-\nu}(x), 2 \leqslant n \leqslant d,
$$

and the regularity conditions $\gamma_{m+1}^{0} \neq 0, m \geqslant 0$.

The 2-orthogonal monic Chebyshev polynomial (2-classical) of first the kind $\left\{\widehat{T}_{n}\right\}_{n \geqslant 0}$ studied in [8], and defined by the next relations where $\alpha$ and $\gamma$ are constants (see also [14])

$$
\left\{\begin{array}{l}
\widehat{T}_{0}(x)=1, \widehat{T}_{1}(x)=x, \widehat{T}_{2}(x)=x^{2}-\alpha \\
\widehat{T}_{n+3}(x)=x \widehat{T}_{n+2}(x)-\alpha \widehat{T}_{n+1}(x)-\gamma \widehat{T}_{n}(x), n \geqslant 0, \gamma \neq 0 .
\end{array}\right.
$$

Definition 1. An d-orthogonal polynomial sequence $\left\{P_{n}\right\}_{n \geqslant 0}$ is called $d$ classical d-orthogonal polynomial sequence if both $\left\{P_{n}\right\}_{n \geqslant 0}$ and its derivative $\left\{P_{n}^{\prime}\right\}_{n \geqslant 0}$ are $d$-orthogonal.

Note that is the 2-classical 2-orthogonal polynomial sequence analougous to the Chebyshev orthogonal polynomial sequence of the first kind $\left\{\widehat{T}_{n}\right\}_{n \geqslant 0}($ see $[8])$.

Lemma 1. [1]. For $n \in \mathbb{N}$, the generating function of the monic 2orthogonal Chebyshev polynomial sequence is given by

$$
\sum_{n=0}^{+\infty} \widehat{T}_{n}(x) z^{n}=\frac{1}{1-x z+\alpha z^{2}+\gamma z^{3}} .
$$

In this paper, we use the new generating function of the 2-orthogonal monic Chebyshev polynomial sequence (2-classical) to give some new generating functions related to the product of the 2-orthogonal Chebyshev polynomials and $k$-Fibonacci numbers, $k$-Pell and $k$-Jacobsthal numbers. We also, use this to derive some new symmetric properties of the generating function of the product of the 2-orthogonal Chebyshev polynomials and other Chebyshev polynomials. 
2. Generating and symmetric functions. Now, we need to introduce a new symmetric function and we give some properties related to this function. We also, give some more useful definitions from the literature which are used in the subsequent sections.

We shall handle functions on different sets of indeterminates (called alphabets, though we shall mostly use commutative indeterminates for the moment). A symmetric function of an alphabet $A$ is a function of the letters which is invariant under permutation of the letters of $A$. Taking an extra indeterminate $z$, one has two fundamental series

$$
\lambda_{z}(A)=\prod_{a \in A}(1+z a), \sigma_{z}(A)=\frac{1}{\prod_{a \in A}(1-z a)} .
$$

The expansion of which gives the elementary symmetric functions $\Lambda_{n}(A)$ and the complete functions $S_{n}(A)$ :

$$
\lambda_{z}(A)=\sum_{n=0}^{\infty} \Lambda_{n}(A) z^{n}, \sigma_{z}(A)=\sum_{n=0}^{\infty} S_{n}(A) z^{n} .
$$

Start with the following definitions.

Definition 2. Let $A$ and $B$ be any two alphabets, then we give $S_{n}(A-B)$ by the following form

$$
\frac{\prod_{b \in B}(1-z b)}{\prod_{a \in A}(1-z a)}=\sum_{n=0}^{\infty} S_{n}(A-B) z^{n}=\sigma_{z}(A-B),
$$

with the condition $S_{n}(A-B)=0$ for $n<0$ [2].

Taking $A=\{0,0, \ldots, 0\}$ in (3) gives

$$
\prod_{b \in B}(1-z b)=\sum_{n=0}^{\infty} S_{n}(-B) z^{n}=\lambda_{z}(-B) .
$$

Further, in the case $A=\{0,0, \ldots, 0\}$ or $B=\{0,0, \ldots, 0\}$, we have

$$
\sum_{n=0}^{\infty} S_{n}(A-B) z^{n}=\sigma_{z}(A) \times \lambda_{z}(-B) .
$$


Thus,

$$
S_{n}(A-B)=\sum_{k=0}^{n} S_{n-k}(A) S_{k}(-B) .
$$

Definition 3. [3] Let $g$ be any function on $\mathbb{R}^{n}$, then we consider the divided difference operator as the following form

$$
\begin{gathered}
\partial_{x_{i} x_{i+1}}(g)= \\
=\frac{g\left(x_{1}, \ldots, x_{i}, x_{i+1}, \ldots, x_{n}\right)-g\left(x_{1}, \ldots, x_{i-1}, x_{i+1}, x_{i}, x_{i+2}, \ldots, x_{n}\right)}{x_{i}-x_{i+1}} .
\end{gathered}
$$

Definition 4. [4] Given an alphabet $E=\left\{e_{1}, e_{2}\right\}$, the symmetrizing operator $\delta_{e_{1} e_{2}}^{k}$ is defined by

$$
\delta_{e_{1} e_{2}}^{k}\left(e_{1}^{n}\right)=\frac{e_{1}^{k+n}-e_{2}^{k+n}}{e_{1}-e_{2}}=S_{k+n-1}\left(e_{1}+e_{2}\right), k, n \geqslant 0 .
$$

In this part, the following lemmas is one of the key tools of the proof of our main results.

Lemma 2. [2] Given an alphabet $E=\left\{e_{1}, e_{2}, e_{3}\right\}$, we have

$$
\sum_{n=0}^{\infty} S_{n}(E) z^{n}=\frac{1}{\left(1-e_{1} z\right)\left(1-e_{2} z\right)\left(1-e_{3} z\right)}
$$

with

$$
\begin{gathered}
\left(1-e_{1} z\right)\left(1-e_{2} z\right)\left(1-e_{3} z\right)= \\
=1-\left(e_{1}+e_{2}+e_{3}\right) z+\left(e_{1} e_{2}+e_{1} e_{3}+e_{2} e_{3}\right) z^{2}-e_{1} e_{2} e_{3} z^{3}= \\
=1+S_{1}(-E) z+S_{2}(-E) z^{2}+S_{3}(-E) z^{3} .
\end{gathered}
$$

Lemma 3. [2] Given two alphabets $E=\left\{e_{1}, e_{2}, e_{3}\right\}$ and $A=\left\{a_{1},-a_{2}\right\}$, we have

$$
\sum_{n=0}^{\infty} S_{n}(E) S_{n}(A) z^{n}=\frac{1+a_{1} a_{2} S_{2}(-E) z^{2}+a_{1} a_{2}\left(a_{1}-a_{2}\right) S_{3}(-E) z^{3}}{\prod_{e \in E}\left(1-e a_{1} z\right) \prod_{e \in E}\left(1+e a_{2} z\right)} .
$$

Lemma 4. [2] Let $A=\left\{a_{1},-a_{2}\right\}$ and $E=\left\{e_{1}, e_{2}, e_{3}\right\}$ two alphabets, we have

$$
\sum_{n=0}^{\infty} S_{n}(E) S_{n-1}(A) z^{n}=
$$




$$
=\frac{-S_{1}(-E) z-\left(a_{1}-a_{2}\right) S_{2}(-E) z^{2}-\left(\left(a_{1}-a_{2}\right)^{2}+a_{1} a_{2}\right) S_{3}(-E) z^{3}}{\prod_{e \in E}\left(1-e a_{1} z\right) \prod_{e \in E}\left(1+e a_{2} z\right)} .
$$

Note that, the substitution of $\left\{\begin{array}{l}S_{1}(-E)=-x \\ S_{2}(-E)=\alpha \\ S_{3}(-E)=\gamma\end{array}\right.$, in (7) gives the following result:

$$
\sum_{n=0}^{\infty} S_{n}(E) z^{n}=\frac{1}{1-x z+\alpha z^{2}+\gamma z^{3}}=\sum_{n=0}^{+\infty} \widehat{T}_{n}(x) z^{n},
$$

which represents the generating function of 2-orthogonal monic Chebyshev polynomials, with $S_{n}(E)=\widehat{T}_{n}(x)$, (see [1]).

3. Generating functions of binary products of 2-Chebyshev polynomials and numbers. In this section, we are going to create the new generating functions of products of 2-Chebyshev polynomials and some numbers ( $k$-Fibonacci, $k$-Pell and $k$-Jacobsthal) based on Lemmas 2,3 and 4 .

Theorem 1. For $n \in \mathbb{N}$, the generating function of the product of 2orthogonal Chebyshev polynomial and $k$-Fibonacci numbers is given by

$$
\sum_{n=0}^{+\infty} \widehat{T}_{n}(x) F_{k, n} z^{n}=\frac{1+\alpha z^{2}+k \gamma z^{3}}{f_{1}(z)}
$$

with

$$
\begin{aligned}
f_{1}(z) & =1-k x z+\left(\left(k^{2}+2\right) \alpha-x^{2}\right) z^{2}+\left(\left(k^{2}+3\right) k \gamma+k \alpha x\right) z^{3}+ \\
& +\left(\left(k^{2}+2\right) \gamma x+\alpha^{2}\right) z^{4}+k \alpha \gamma z^{5}-\gamma^{2} z^{6} .
\end{aligned}
$$

Proof. By [6], we have $F_{k, n}=S_{n}\left(a_{1}+\left[-a_{2}\right]\right)$. Then, we can see that

$$
\begin{aligned}
& \sum_{n=0}^{\infty} \widehat{T}_{n}(x) F_{k, n} z^{n}=\sum_{n=0}^{\infty} S_{n}(E) S_{n}\left(a_{1}+\left[-a_{2}\right]\right) z^{n}= \\
= & \frac{1}{\left(a_{1}+a_{2}\right)}\left(a_{1} \sum_{n=0}^{\infty} S_{n}(E)\left(a_{1} z\right)^{n}+a_{2} \sum_{n=0}^{\infty} S_{n}(E)\left(-a_{2} z\right)^{n}\right) .
\end{aligned}
$$

In addition, we have

$$
\sum_{n=0}^{\infty} S_{n}(E)\left(a_{1} z\right)^{n}=\frac{1}{1+S_{1}(-E) a_{1} z+S_{2}(-E) a_{1}^{2} z^{2}+S_{3}(-E) a_{1}^{3} z^{3}},
$$


and

$$
\sum_{n=0}^{\infty} S_{n}(E)\left(-a_{2} z\right)^{n}=\frac{1}{1-S_{1}(-E) a_{2} z+S_{2}(-E) a_{2}^{2} z^{2}-S_{3}(-E) a_{2}^{3} z^{3}} .
$$

According to Lemma 2, we obtain

$$
\begin{aligned}
\sum_{n=0}^{\infty} \widehat{T}_{n}(x) F_{k, n} z^{n}= & \frac{1}{a_{1}+a_{2}}\left(\frac{a_{1}}{1+S_{1}(-E) a_{1} z+S_{2}(-E) a_{1}^{2} z^{2}+S_{3}(-E) a_{1}^{3} z^{3}}+\right. \\
& \left.+\frac{a_{2}}{1-S_{1}(-E) a_{2} z+S_{2}(-E) a_{2}^{2} z^{2}-S_{3}(-E) a_{2}^{3} z^{3}}\right)
\end{aligned}
$$

Then, by reduce to same denominator, we obtain the following result

$$
\sum_{n=0}^{\infty} \widehat{T}_{n}(x) F_{k, n} z^{n}=\frac{1+p_{1}(x) z^{2}+p_{2}(x) z^{3}}{1+q_{1}(x) z+q_{2}(x) z^{2}+q_{3}(x) z^{3}+q_{4}(x) z^{4}+q_{5}(x) z^{5}+q_{6}(x) z^{6}},
$$

where

$$
\begin{gathered}
p_{1}(x)=a_{1} a_{2} S_{2}(-E), p_{2}(x)=a_{1} a_{2}\left(a_{1}-a_{2}\right) S_{3}(-E), q_{1}(x)=\left(a_{1}-a_{2}\right) S_{1}(-E), \\
q_{2}(x)=S_{2}(-E)\left(a_{1}-a_{2}\right)^{2}-a_{1} a_{2}\left(S_{1}(-E)^{2}-2 S_{2}(-E)\right), \\
q_{3}(x)=S_{3}(-E)\left(a_{1}-a_{2}\right)^{3}-a_{1} a_{2}\left(a_{1}-a_{2}\right)\left(S_{1}(-E) S_{2}(-E)-3 S_{3}(-E)\right), \\
q_{4}(x)=-a_{1} a_{2}\left(a_{1}-a_{2}\right)^{2} S_{3}(-E) S_{1}(-E)+a_{1}^{2} a_{2}^{2}\left(S_{2}(-E)^{2}-2 S_{3}(-E) S_{1}(-E)\right), \\
q_{5}(x)=a_{1}^{2} a_{2}^{2} S_{3}(-E) S_{2}(-E)\left(a_{1}-a_{2}\right), \quad q_{6}(x)=-S_{3}(-E)^{2} a_{1}^{3} a_{2}^{3} .
\end{gathered}
$$

After a simple calculation, of $p_{i}(x)$ and $q_{i}(x)$, we obtain the desired result.

Theorem 2. For $n \in \mathbb{N}$, the generating function of the product between 2-orthogonal Chebyshev polynomials and $k$-Pell numbers is given by

$$
\sum_{n=0}^{+\infty} \widehat{T}_{n}(x) P_{k, n} z^{n}=\frac{x z-2 \alpha z^{2}-\gamma(4+k) z^{3}}{f_{2}(z)}
$$

with

$$
\begin{aligned}
f_{2}(z) & =1-2 x z+\left(2(k+2) \alpha-k x^{2}\right) z^{2}+(2 \gamma(3 k+4)+2 k \alpha x) z^{3}+ \\
& +\left(2 k(k+2) \gamma x+k^{2} \alpha^{2}\right) z^{4}+2 k^{2} \alpha \gamma z^{5}-k^{3} \gamma^{2} z^{6} .
\end{aligned}
$$


Proof. By referred to [6], we have $P_{k, n}=S_{n-1}\left(a_{1}+\left[-a_{2}\right]\right)$. On the other hand, we can see that

$$
\begin{aligned}
\sum_{n=0}^{\infty} \widehat{T}_{n}(x) P_{k, n} z^{n} & =\sum_{n=0}^{\infty} S_{n}(E) S_{n-1}\left(a_{1}+\left[-a_{2}\right]\right) z^{n}= \\
& =\frac{1}{\left(a_{1}+a_{2}\right)}\left(\sum_{n=0}^{\infty} S_{n}(E)\left(a_{1} z\right)^{n}-\sum_{n=0}^{\infty} S_{n}(E)\left(-a_{2} z\right)^{n}\right) .
\end{aligned}
$$

Using the Lemma 3, we can write

$$
\begin{aligned}
\sum_{n=0}^{\infty} \widehat{T}_{n}(x) P_{k, n} z^{n} & =\frac{1}{a_{1}+a_{2}}\left(\frac{1}{1+S_{1}(-E) a_{1} z+S_{2}(-E) a_{1}^{2} z^{2}+S_{3}(-E) a_{1}^{3} z^{3}}-\right. \\
& \left.-\frac{1}{1-S_{1}(-E) a_{2} z+S_{2}(-E) a_{2}^{2} z^{2}-S_{3}(-E) a_{2}^{3} z^{3}}\right) .
\end{aligned}
$$

Equivalently

$$
\sum_{n=0}^{\infty} \widehat{T}_{n}(x) P_{k, n} z^{n}=\frac{p_{1}(x) z+p_{2}(x) z^{2}+p_{3}(x) z^{3}}{1+q_{1}(x) z+q_{2}(x) z^{2}+q_{3}(x) z^{3}+q_{4}(x) z^{4}+q_{5}(x) z^{5}+q_{6}(x) z^{6}},
$$

where

$$
\begin{gathered}
p_{1}(x)=-S_{1}(-E), \quad p_{2}(x)=-\left(a_{1}-a_{2}\right) S_{2}(-E), \\
p_{3}(x)=-\left(\left(a_{1}-a_{2}\right)^{2}+a_{1} a_{2}\right) S_{3}(-E), \quad q_{1}(x)=\left(a_{1}-a_{2}\right) S_{1}(-E), \\
q_{2}(x)=S_{2}(-E)\left(a_{1}-a_{2}\right)^{2}-a_{1} a_{2}\left(S_{1}(-E)^{2}-2 S_{2}(-E)\right), \\
q_{3}(x)=S_{3}(-E)\left(a_{1}-a_{2}\right)^{3}-a_{1} a_{2}\left(a_{1}-a_{2}\right)\left(S_{1}(-E) S_{2}(-E)-3 S_{3}(-E)\right), \\
q_{4}(x)=a_{1} a_{2}\left(a_{1}-a_{2}\right)^{2} S_{3}(-E) S_{1}(-E)-a_{1}^{2} a_{2}^{2}\left(S_{2}(-E)^{2}-2 S_{3}(-E) S_{1}(-E)\right), \\
q_{5}(x)=a_{1}^{2} a_{2}^{2} S_{3}(-E) S_{2}(-E)\left(a_{1}-a_{2}\right), \quad q_{6}(x)=-S_{3}(-E)^{2} a_{1}^{3} a_{2}^{3} .
\end{gathered}
$$

This gives, after a simple calculation, the following

$$
\begin{gathered}
p_{1}(x)=x, p_{2}(x)=-2 \alpha, p_{3}(x)=-\gamma(4+k), q_{1}(x)=-2 x, q_{2}(x)=2(k+2) \alpha-k x^{2}, \\
q_{3}(x)=2 \gamma(3 k+4)+2 k \alpha x, q_{4}(x)=2 k(k+2) \gamma x+k^{2} \alpha^{2}, \\
q_{5}(x)=2 k^{2} \alpha \gamma, \quad q_{6}(x)=-k^{3} \gamma^{2} .
\end{gathered}
$$

Hence, the Theorem 2 is valid. 
Theorem 3. For $n \in \mathbb{N}$, the generating function of the product of 2-orthogonal Chebyshev polynomials and $k$-Jacobsthal numbers is given by

$$
\sum_{n=0}^{+\infty} \widehat{T}_{n}(x) J_{k, n} z^{n}=\frac{x z-k \alpha z^{2}-\gamma\left(2+k^{2}\right) z^{3}}{f_{3}(z)}
$$

with

$$
\begin{aligned}
f_{3}(z) & =1-k x z+\left(\left(k^{2}+4\right) \alpha-2 x^{2}\right) z^{2}+\left(k \gamma\left(k^{2}+6\right)+2 k \alpha x\right) z^{3}+ \\
& +\left(2\left(k^{2}+4\right) \gamma x+4 \alpha^{2}\right) z^{4}+4 k \alpha \gamma z^{5}-8 \gamma^{2} z^{6} .
\end{aligned}
$$

Proof. Recall that, we have $J_{k, n}=S_{n-1}\left(a_{1}+\left[-a_{2}\right]\right)$ (see [6]). We see that

$$
\begin{aligned}
\sum_{n=0}^{\infty} \widehat{T}_{n}(x) J_{k, n} z^{n} & =\sum_{n=0}^{\infty} S_{n}(E) S_{n-1}\left(a_{1}+\left[-a_{2}\right]\right) z^{n}= \\
& =\frac{1}{\left(a_{1}+a_{2}\right)}\left(\sum_{n=0}^{\infty} S_{n}(E)\left(a_{1} z\right)^{n}-\sum_{n=0}^{\infty} S_{n}(E)\left(-a_{2} z\right)^{n}\right)
\end{aligned}
$$

According to Lemma 4, this gives the following equality

$$
\begin{aligned}
\sum_{n=0}^{\infty} \widehat{T}_{n}(x) J_{k, n} z^{n}= & \frac{1}{a_{1}+a_{2}}\left(\frac{1}{1+S_{1}(-E) a_{1} z+S_{2}(-E) a_{1}^{2} z^{2}+S_{3}(-E) a_{1}^{3} z^{3}}-\right. \\
& \left.-\frac{1}{1-S_{1}(-E) a_{2} z+S_{2}(-E) a_{2}^{2} z^{2}-S_{3}(-E) a_{2}^{3} z^{3}}\right)
\end{aligned}
$$

Then, by reduce to same denominator, we get

$$
\sum_{n=0}^{\infty} \widehat{T}_{n}(x) J_{k, n} z^{n}=\frac{p_{1}(x) z+p_{2}(x) z^{2}+p_{3}(x) z^{3}}{1+q_{1}(x) z+q_{2}(x) z^{2}+q_{3}(x) z^{3}+q_{4}(x) z^{4}+q_{5}(x) z^{5}+q_{6}(x) z^{6}},
$$

where

$$
\begin{gathered}
p_{1}(x)=-S_{1}(-E), p_{2}(x)=-\left(a_{1}-a_{2}\right) S_{2}(-E), \\
p_{3}(x)=-\left(\left(a_{1}-a_{2}\right)^{2}+a_{1} a_{2}\right) S_{3}(-E), q_{1}(x)=\left(a_{1}-a_{2}\right) S_{1}(-E), \\
q_{2}(x)=S_{2}(-E)\left(a_{1}-a_{2}\right)^{2}-a_{1} a_{2}\left(S_{1}(-E)^{2}-2 S_{2}(-E)\right), \\
q_{3}(x)=S_{3}(-E)\left(a_{1}-a_{2}\right)^{3}-a_{1} a_{2}\left(a_{1}-a_{2}\right)\left(S_{1}(-E) S_{2}(-E)-3 S_{3}(-E)\right), \\
q_{4}(x)=a_{1} a_{2}\left(a_{1}-a_{2}\right)^{2} S_{3}(-E) S_{1}(-E)-a_{1}^{2} a_{2}^{2}\left(S_{2}(-E)^{2}-2 S_{3}(-E) S_{1}(-E)\right),
\end{gathered}
$$




$$
q_{5}(x)=a_{1}^{2} a_{2}^{2} S_{3}(-E) S_{2}(-E)\left(a_{1}-a_{2}\right), q_{6}(x)=-S_{3}(-E)^{2} a_{1}^{3} a_{2}^{3} .
$$

This gives, after a simple calculation, the following

$$
\begin{gathered}
p_{1}(x)=x, p_{2}(x)=-k \alpha, p_{3}(x)=-\gamma\left(2+k^{2}\right), q_{1}(x)=-k x, \\
q_{2}(x)=\left(k^{2}+4\right) \alpha-2 x^{2}, q_{3}(x)=k \gamma\left(k^{2}+6\right)+2 k \alpha x, \\
q_{4}(x)=2\left(k^{2}+4\right) \gamma x+4 \alpha^{2}, q_{5}(x)=4 k \alpha \gamma, q_{6}(x)=-8 \gamma^{2} .
\end{gathered}
$$

Hence, the Theorem 3 is valid.

4. Generating functions of binary products of 2-Chebyshev polynomials and other Chebyshev polynomials. The following lemmas (see [2]) are the key tools of the proof of our main results.

Lemma 5. Given two alphabets $E=\left\{e_{1}, e_{2}, e_{3}\right\}$ and $A=\left\{2 a_{1},-2 a_{2}\right\}$, we have

$$
\sum_{n=0}^{\infty} S_{n}(E) S_{n}(A) z^{n}=\frac{1+4 a_{1} a_{2} S_{2}(-E) z^{2}+8 a_{1} a_{2}\left(a_{1}-a_{2}\right) S_{3}(-E) z^{3}}{\prod_{e \in E}\left(1-2 e a_{1} z\right) \prod_{e \in E}\left(1+2 e a_{2} z\right)} .
$$

Lemma 6. Let $A=\left\{2 a_{1},-2 a_{2}\right\}$ and $E=\left\{e_{1}, e_{2}, e_{3}\right\}$ two alphabets, we have

$$
\begin{gathered}
\sum_{n=0}^{\infty} S_{n}(E) S_{n-1}(A) z^{n}= \\
=\frac{-S_{1}(-E) z-2\left(a_{1}-a_{2}\right) S_{2}(-E) z^{2}-4\left(\left(a_{1}-a_{2}\right)^{2}+a_{1} a_{2}\right) S_{3}(-E) z^{3}}{\prod_{e \in E}\left(1-2 e a_{1} z\right) \prod_{e \in E}\left(1+2 e a_{2} z\right)} .
\end{gathered}
$$

Based on the last lemmas, we can state the following theorems which represent the new generating functions of products of 2-Chebyshev polynomials of the first kind with the other Chebyshev polynomials.

Theorem 4. For $n \in \mathbb{N}$, the generating function of the product of 2-orthogonal Chebyshev polynomials and monic chebyshev polynomials of the second kind is given by

$$
\sum_{n=0}^{+\infty} \widehat{T}_{n}(x) U_{n}(y) z^{n}=\frac{1-\alpha z^{2}-2 \gamma y z^{3}}{f_{4}(z)}
$$

with 


$$
\begin{array}{r}
f_{4}(z)=1-2 x y z+\left(2\left(2 y^{2}-1\right) \alpha+x^{2}\right) z^{2}+\left(2\left(4 y^{2}-3\right) \gamma y-2 \alpha x y\right) z^{3}- \\
-\left(2\left(2 y^{2}-1\right) \gamma x-\alpha^{2}\right) z^{4}+2 \alpha \gamma y z^{5}+\gamma^{2} z^{6} .
\end{array}
$$

Proof. By using [5], we have $U_{n}(y)=S_{n}\left(2 a_{1}+\left[-2 a_{2}\right]\right)$. Then, we can easily seen that

$$
\begin{gathered}
\sum_{n=0}^{\infty} \widehat{T}_{n}(x) U_{n}(y) z^{n}=\sum_{n=0}^{\infty} S_{n}(E) S_{n}\left(2 a_{1}+\left[-2 a_{2}\right]\right) z^{n}= \\
=\frac{1}{2\left(a_{1}+a_{2}\right)}\left(2 a_{1} \sum_{n=0}^{\infty} S_{n}(E)\left(2 a_{1} z\right)^{n}+2 a_{2} \sum_{n=0}^{\infty} S_{n}(E)\left(-2 a_{2} z\right)^{n}\right)= \\
=\frac{1}{2\left(a_{1}+a_{2}\right)}\left(\frac{2 a_{1}}{1+2 S_{1}(-E) a_{1} z+4 S_{2}(-E) a_{1}^{2} z^{2}+8 S_{3}(-E) a_{1}^{3} z^{3}}+\right. \\
\left.\quad+\frac{2 a_{2}}{1-2 S_{1}(-E) a_{2} z+4 S_{2}(-E) a_{2}^{2} z^{2}-8 S_{3}(-E) a_{2}^{3} z^{3}}\right),
\end{gathered}
$$

by using the Lemma 5 . Equivalently, we get

$$
\sum_{n=0}^{\infty} \widehat{T}_{n}(x) U_{n}(y) z^{n}=\frac{1+f_{1}(x) z^{2}+f_{2}(x) z^{3}}{1+g_{1}(x) z+g_{2}(x) z^{2}+g_{3}(x) z^{3}-g_{4}(x) z^{4}+g_{5}(x) z^{5}-g_{6}(x) z^{6}},
$$

where

$$
\begin{gathered}
f_{1}(x)=4 a_{1} a_{2} S_{2}(-E), f_{2}(x)=8 a_{1} a_{2}\left(a_{1}-a_{2}\right) S_{3}(-E), g_{1}(x)=2\left(a_{1}-a_{2}\right) S_{1}(-E), \\
g_{2}(x)=4 S_{2}(-E)\left(a_{1}-a_{2}\right)^{2}-4 a_{1} a_{2}\left(S_{1}(-E)^{2}-2 S_{2}(-E)\right), \\
g_{3}(x)=8 S_{3}(-E)\left(a_{1}-a_{2}\right)^{3}-8 a_{1} a_{2}\left(a_{1}-a_{2}\right)\left(S_{1}(-E) S_{2}(-E)-3 S_{3}(-E)\right), \\
g_{4}(x)=16 a_{1} a_{2}\left(a_{1}-a_{2}\right)^{2} S_{3}(-E) S_{1}(-E)-16 a_{1}^{2} a_{2}^{2}\left(S_{2}(-E)^{2}-2 S_{3}(-E) S_{1}(-E)\right), \\
g_{5}(x)=32 a_{1}^{2} a_{2}^{2} S_{3}(-E) S_{2}(-E)\left(a_{1}-a_{2}\right), \quad g_{6}(x)=64 S_{3}(-E)^{2} a_{1}^{3} a_{2}^{3} .
\end{gathered}
$$

After a simple calculation of $f_{i}(x)$ and $g_{i}(x)$ we obtain the desired result.

Theorem 5. For $n \in \mathbb{N}$, the generating function of the product of 2orthogonal Chebyshev polynomials and Chebyshev polynomials of the first kind is given by

$$
\sum_{n=0}^{+\infty} \widehat{T}_{n}(x) T_{n}(y) z^{n}=\frac{1-x y z+\alpha\left(2 y^{2}-1\right) z^{2}+\gamma\left(4 y^{2}-3\right) y z^{3}}{f_{5}(z)},
$$


with

$$
\begin{aligned}
f_{5}(z) & =1-2 x y z+\left(2\left(2 y^{2}-1\right) \alpha+x^{2}\right) z^{2}+\left(2\left(4 y^{2}-3\right) \gamma y-2 \alpha x y\right) z^{3}- \\
& -\left(2\left(2 y^{2}-1\right) \gamma x-\alpha^{2}\right) z^{4}+2 \alpha \gamma y z^{5}+\gamma^{2} z^{6}
\end{aligned}
$$

Proof. By [5], we have $T_{n}(y)=S_{n}\left(2 a_{1}+\left[-2 a_{2}\right]\right)-y S_{n-1}\left(2 a_{1}+\left[-2 a_{2}\right]\right)$. In addition, we can see that

$$
\begin{aligned}
& \sum_{n=0}^{\infty} \widehat{T}_{n}(x) T_{n}(y) z^{n}= \\
& \quad \sum_{n=0}^{\infty} S_{n}(E)\left(S_{n}\left(2 a_{1}+\left[-2 a_{2}\right]\right)-y S_{n-1}\left(2 a_{1}+\left[-2 a_{2}\right]\right)\right) z^{n}= \\
& =\sum_{n=0}^{\infty} S_{n}(E) S_{n}\left(2 a_{1}+\left[-2 a_{2}\right]\right) z^{n}-y \sum_{n=0}^{\infty} S_{n}(E) S_{n-1}\left(2 a_{1}+\left[-2 a_{2}\right]\right)= \\
& =\sum_{n=0}^{\infty} \widehat{T}_{n}(x) U_{n}(y) z^{n}-\frac{y}{2\left(a_{1}+a_{2}\right)}\left(\sum_{n=0}^{\infty} S_{n}(E)\left(2 a_{1} z\right)^{n}-\sum_{n=0}^{\infty} S_{n}(E)\left(-2 a_{2} z\right)^{n}\right),
\end{aligned}
$$

which gives

$$
\begin{gathered}
\sum_{n=0}^{\infty} \widehat{T}_{n}(x) T_{n}(y) z^{n}=\sum_{n=0}^{\infty} \widehat{T}_{n}(x) U_{n}(y) z^{n}- \\
-\frac{y}{2\left(a_{1}+a_{2}\right)}\left(\frac{1}{1+2 S_{1}(-E) a_{1} z+4 S_{2}(-E) a_{1}^{2} z^{2}+8 S_{3}(-E) a_{1}^{3} z^{3}}-\right. \\
\left.-\frac{1}{1-2 S_{1}(-E) a_{2} z+4 S_{2}(-E) a_{2}^{2} z^{2}-8 S_{3}(-E) a_{2}^{3} z^{3}}\right)
\end{gathered}
$$

After reduce to same denominator, we obtain the following result

$$
\begin{gathered}
\sum_{n=0}^{\infty} \widehat{T}_{n}(x) T_{n}(y) z^{n}=\sum_{n=0}^{\infty} \widehat{T}_{n}(x) U_{n}(y) z^{n}- \\
-y\left(\frac{f_{1}(x) z-f_{2}(x) z^{2}-f_{3}(x) z^{3}}{1+g_{1}(x) z+g_{2}(x) z^{2}+g_{3}(x) z^{3}-g_{4}(x) z^{4}+g_{5}(x) z^{5}-g_{6}(x) z^{6}}\right)
\end{gathered}
$$

where

$$
\begin{gathered}
f_{1}(x)=-S_{1}(-E), f_{2}(x)=2\left(a_{1}-a_{2}\right) S_{2}(-E), \\
f_{3}(x)=4\left(\left(a_{1}-a_{2}\right)^{2}+a_{1} a_{2}\right) S_{3}(-E), g_{1}(x)=2\left(a_{1}-a_{2}\right) S_{1}(-E),
\end{gathered}
$$




$$
\begin{gathered}
g_{2}(x)=4 S_{2}(-E)\left(a_{1}-a_{2}\right)^{2}-4 a_{1} a_{2}\left(S_{1}(-E)^{2}-2 S_{2}(-E)\right), \\
g_{3}(x)=8 S_{3}(-E)\left(a_{1}-a_{2}\right)^{3}-8 a_{1} a_{2}\left(a_{1}-a_{2}\right)\left(S_{1}(-E) S_{2}(-E)-3 S_{3}(-E)\right), \\
g_{4}(x)=16 a_{1} a_{2}\left(a_{1}-a_{2}\right)^{2} S_{3}(-E) S_{1}(-E)-16 a_{1}^{2} a_{2}^{2}\left(S_{2}(-E)^{2}-2 S_{3}(-E) S_{1}(-E)\right), \\
g_{5}(x)=32 a_{1}^{2} a_{2}^{2} S_{3}(-E) S_{2}(-E)\left(a_{1}-a_{2}\right), g_{6}(x)=64 S_{3}(E)^{2} a_{1}^{3} a_{2}^{3} .
\end{gathered}
$$

After a simple calculation of $f_{i}(x)$ and $g_{i}(x)$ we obtain the result.

Theorem 6. For $n \in \mathbb{N}$, the generating function of the product between 2-orthogonal Chebyshev and Chebyshev polynomials of the third kind is given by

$$
\sum_{n=0}^{+\infty} \widehat{T}_{n}(x) V_{n}(y) z^{n}=\frac{1-x z+\alpha(2 y-1) z^{2}+\gamma\left(4 y^{2}-2 y-1\right) z^{3}}{f_{6}(z)},
$$

with

$$
\begin{array}{r}
f_{6}(z)=1-2 x y z+\left(2\left(2 y^{2}-1\right) \alpha+x^{2}\right) z^{2}+\left(2\left(4 y^{2}-3\right) \gamma y-2 \alpha x y\right) z^{3}- \\
-\left(2\left(2 y^{2}-1\right) \gamma x-\alpha^{2}\right) z^{4}+2 \alpha \gamma y z^{5}+\gamma^{2} z^{6} .
\end{array}
$$

Proof. By referred to [5], we have

$$
V_{n}(y)=S_{n}\left(2 a_{1}+\left[-2 a_{2}\right]\right)-S_{n-1}\left(2 a_{1}+\left[-2 a_{2}\right]\right) .
$$

By using the Lemma 6, we obtain

$$
\begin{aligned}
& \sum_{n=0}^{\infty} \widehat{T}_{n}(x) V_{n}(y) z^{n}=\sum_{n=0}^{\infty} S_{n}(E)\left(S_{n}\left(2 a_{1}+\left[-2 a_{2}\right]\right)-S_{n-1}\left(2 a_{1}+\left[-2 a_{2}\right]\right)\right) z^{n}= \\
& \quad=\sum_{n=0}^{\infty} S_{n}(E) S_{n}\left(2 a_{1}+\left[-2 a_{2}\right]\right) z^{n}-\sum_{n=0}^{\infty} S_{n}(E) S_{n-1}\left(2 a_{1}+\left[-2 a_{2}\right]\right)= \\
& =\sum_{n=0}^{\infty} \widehat{T}_{n}(x) U_{n}(y) z^{n}-\frac{1}{2\left(a_{1}+a_{2}\right)}\left(\sum_{n=0}^{\infty} S_{n}(E)\left(2 a_{1} z\right)^{n}-\sum_{n=0}^{\infty} S_{n}(E)\left(-2 a_{2} z\right)^{n}\right) .
\end{aligned}
$$

Equivalently

$$
\begin{aligned}
& \sum_{n=0}^{\infty} \widehat{T}_{n}(x) V_{n}(y) z^{n}=\sum_{n=0}^{\infty} \widehat{T}_{n}(x) U_{n}(y) z^{n}- \\
& \quad-\frac{1}{2\left(a_{1}+a_{2}\right)}\left(\frac{1}{1+2 S_{1}(-E) a_{1} z+4 S_{2}(-E) a_{1}^{2} z^{2}+8 S_{3}(-E) a_{1}^{3} z^{3}}-\right.
\end{aligned}
$$




$$
\left.-\frac{1}{1-2 S_{1}(-E) a_{2} z+4 S_{2}(-E) a_{2}^{2} z^{2}-8 S_{3}(-E) a_{2}^{3} z^{3}}\right),
$$

which gives, after reduce to same denominator, the following result

$$
\begin{aligned}
& \sum_{n=0}^{\infty} \widehat{T}_{n}(x) V_{n}(y) z^{n}=\sum_{n=0}^{\infty} \widehat{T}_{n}(x) U_{n}(y) z^{n}- \\
& \quad-\frac{f_{1}(x) z-f_{2}(x) z^{2}-f_{3}(x) z^{3}}{1+g_{1}(x) z+g_{2}(x) z^{2}+g_{3}(x) z^{3}-g_{4}(x) z^{4}+g_{5}(x) z^{5}-g_{6}(x) z^{6}}
\end{aligned}
$$

where

$$
\begin{gathered}
f_{1}(x)=-S_{1}(-E), f_{2}(x)=2\left(a_{1}-a_{2}\right) S_{2}(-E), \\
f_{3}(x)=4\left(\left(a_{1}-a_{2}\right)^{2}+a_{1} a_{2}\right) S_{3}(-E), g_{1}(x)=2\left(a_{1}-a_{2}\right) S_{1}(-E), \\
g_{2}(x)=4 S_{2}(-E)\left(a_{1}-a_{2}\right)^{2}-4 a_{1} a_{2}\left(S_{1}(-E)^{2}-2 S_{2}(-E)\right), \\
g_{3}(x)=8 S_{3}(-E)\left(a_{1}-a_{2}\right)^{3}-8 a_{1} a_{2}\left(a_{1}-a_{2}\right)\left(S_{1}(-E) S_{2}(-E)-3 S_{3}(-E)\right), \\
g_{4}(x)=16 a_{1} a_{2}\left(a_{1}-a_{2}\right)^{2} S_{3}(-E) S_{1}(-E)-16 a_{1}^{2} a_{2}^{2}\left(S_{2}(-E)^{2}-2 S_{3}(-E) S_{1}(-E)\right), \\
g_{5}(x)=32 a_{1}^{2} a_{2}^{2} S_{3}(-E) S_{2}(-E)\left(a_{1}-a_{2}\right), g_{6}(x)=64 S_{3}(-E)^{2} a_{1}^{3} a_{2}^{3} .
\end{gathered}
$$

Hence, after a simple calculation of $f_{i}(x)$ and $g_{i}(x)$, we can obtain the result.

Theorem 7. For $n \in \mathbb{N}$, the generating function of the product between 2-orthogonal Chebyshev and Chebyshev polynomials of the fourth kind is given by

$$
\sum_{n=0}^{+\infty} \widehat{T}_{n}(x) W_{n}(y) z^{n}=\frac{1+x z-\alpha(2 y+1) z^{2}-\gamma\left(4 y^{2}+2 y-1\right) z^{3}}{f_{7}(z)}
$$

with

$$
\begin{array}{r}
\left.f_{7}(z)=1-2 x y z+\left(2\left(2 y^{2}-1\right) \alpha+x^{2}\right) z^{2}+2\left(4 y^{2}-3\right) \gamma y-2 \alpha x y\right) z^{3}- \\
-\left(2\left(2 y^{2}-1\right) \gamma x-\alpha^{2}\right) z^{4}+2 \alpha \gamma y z^{5}+\gamma^{2} z^{6}
\end{array}
$$

Proof. According to [5], we have

$$
W_{n}(y)=S_{n}\left(2 a_{1}+\left[-2 a_{2}\right]\right)+S_{n-1}\left(2 a_{1}+\left[-2 a_{2}\right]\right) .
$$


We, easily, see that

$$
\begin{aligned}
& \sum_{n=0}^{\infty} \widehat{T}_{n}(x) W_{n}(y) z^{n}=\sum_{n=0}^{\infty} S_{n}(E)\left(S_{n}\left(2 a_{1}+\left[-2 a_{2}\right]\right)+S_{n-1}\left(2 a_{1}+\left[-2 a_{2}\right]\right)\right) z^{n}= \\
& \quad=\sum_{n=0}^{\infty} S_{n}(E) S_{n}\left(2 a_{1}+\left[-2 a_{2}\right]\right) z^{n}+\sum_{n=0}^{\infty} S_{n}(E) S_{n-1}\left(2 a_{1}+\left[-2 a_{2}\right]\right)= \\
& =\sum_{n=0}^{\infty} \widehat{T}_{n}(x) U_{n}(y) z^{n}+\frac{1}{2\left(a_{1}+a_{2}\right)}\left(\sum_{n=0}^{\infty} S_{n}(E)\left(2 a_{1} z\right)^{n}-\sum_{n=0}^{\infty} S_{n}(E)\left(-2 a_{2} z\right)^{n}\right),
\end{aligned}
$$

which implies that

$$
\begin{gathered}
\sum_{n=0}^{\infty} \widehat{T}_{n}(x) W_{n}(y) z^{n}=\sum_{n=0}^{\infty} \widehat{T}_{n}(x) U_{n}(y) z^{n}+ \\
+\frac{1}{2\left(a_{1}+a_{2}\right)}\left(\frac{1}{1+2 S_{1}(-E) a_{1} z+4 S_{2}(-E) a_{1}^{2} z^{2}+8 S_{3}(-E) a_{1}^{3} z^{3}}-\right. \\
\left.-\frac{1}{1-2 S_{1}(-E) a_{2} z+4 S_{2}(-E) a_{2}^{2} z^{2}-8 S_{3}(-E) a_{2}^{3} z^{3}}\right),
\end{gathered}
$$

since we have the Lemma 6 . Then, by reduce to same denominator, we get

$$
\begin{gathered}
\sum_{n=0}^{\infty} \widehat{T}_{n}(x) W_{n}(y) z^{n}=\sum_{n=0}^{\infty} \widehat{T}_{n}(x) U_{n}(y) z^{n}+ \\
+\frac{f_{1}(x) z-f_{2}(x) z^{2}-f_{3}(x) z^{3}}{1+g_{1}(x) z+g_{2}(x) z^{2}+g_{3}(x) z^{3}-g_{4}(x) z^{4}+g_{5}(x) z^{5}-g_{6}(x) z^{6}},
\end{gathered}
$$

where

$$
\begin{gathered}
f_{1}(x)=-S_{1}(-E), \quad f_{2}(x)=2\left(a_{1}-a_{2}\right) S_{2}(-E), \\
f_{3}(x)=4\left(\left(a_{1}-a_{2}\right)^{2}+a_{1} a_{2}\right) S_{3}(-E), g_{1}(x)=2\left(a_{1}-a_{2}\right) S_{1}(-E), \\
g_{2}(x)=4 S_{2}(-E)\left(a_{1}-a_{2}\right)^{2}-4 a_{1} a_{2}\left(S_{1}(-E)^{2}-2 S_{2}(-E)\right), \\
g_{3}(x)=8 S_{3}(-E)\left(a_{1}-a_{2}\right)^{3}-8 a_{1} a_{2}\left(a_{1}-a_{2}\right)\left(S_{1}(-E) S_{2}(-E)-3 S_{3}(-E)\right), \\
g_{4}(x)=16 a_{1} a_{2}\left(a_{1}-a_{2}\right)^{2} S_{3}(-E) S_{1}(-E)-16 a_{1}^{2} a_{2}^{2}\left(S_{2}(-E)^{2}-2 S_{3}(-E) S_{1}(-E)\right), \\
g_{5}(x)=32 a_{1}^{2} a_{2}^{2} S_{3}(-E) S_{2}(-E)\left(a_{1}-a_{2}\right), g_{6}(x)=64 S_{3}(-E)^{2} a_{1}^{3} a_{2}^{3} .
\end{gathered}
$$

This gives, after a simple calculation of $f_{i}(x)$ and $g_{i}(x)$, the desired result. 
Conclusion. In this paper, the new theorems has been proposed in order to determine the generating functions. The proposed theorems is based on the symmetric functions. The obtained results agree with the results obtained in some previous works.

Acknowledgment. Sincere thanks are due to the referee for his/her careful reading of the manuscript and for his/her valuable comments. This work was supported by Directorate General for Scientific Research and Technological Development (DGRSDT), Algeria.

\section{References}

[1] Ben Cheikh Y., Ben Romhane N., d-orthogonal polynomials sets of Tchebytchev type, in : Proceedings of the International Conference on Difference Equations, Special Functions and Orthogonal Polynomials, S. Elaydi et al. (Ed.), Munich, Germany, 25-30 July 2005, World Scientific, 100-111. DOI: https://doi.org/10.1142/9789812770752_0008.

[2] Boussayoud A., Boughaba S., On Some Identities and Generating Functions for k-Pell sequences and Chebyshev polynomials. Online J. Anal. Comb., 2019, no. 14, pp. 1-13.

[3] Boussayoud, A. On some identities and generating functions for PellLucas numbers. Online. J. Anal. Comb., 2017, no. 12, pp. 1-10.

[4] Boussayoud A., kerada M., Boulyer M., A simple and accurate method for determination of some generalized sequence of numbers. Int. J. Pure Appl. Math., 2016, no. 108, pp. 503-511.

[5] Boussayoud A., Abderrezzak A., Kerada M., Some applications of symmetric functions. Integers., 2015, no. 15, pp. 1-7.

[6] Boughaba S., Boussayoud A., Kerada M., Construction of Symmetric Functions of Generalized Fibonacci Numbers. Tamap J. Mathematics and Statistics., 2019, no. 3, pp. 1-7.

DOI: https://doi.org/10.29371/2019.16.SI01.

[7] Chihara T.S., An Introduction to Orthogonal Polynomials. Gordon and Breach, New York, 1978.

[8] Douak K., Maroni P., On d-orthogonal Tchebychev polynomials. I. Appl. Numer Math., 1997, no. 24, pp. 23-53.

[9] Kim D.S., Kim T., Lee S.H., Some identities for Bernoulli polynomials involving Chebychev polynomials. J. Comput. Anal. Appl., 2014, no. 16(1), pp. $172-180$. 
[10] Kim D.S., Doglgy D.V., Kim T., Rim S.H., Identities involving Bernoulli and Euler polynomials arising from Chebychev polynomials. Proc. Jangjeon Math. Soc., 2012, no. 15 (4), pp. 361-370.

[11] Maroni P., Une théorie algébrique des polynômes orthogonaux Applications aux polynômes orthogonaux semi-classiques, In Orthogonal Polynomials and their Applications, C. Brezinski et al. Editors, IMACS Ann. Comput. Appl. Math., 1991, no. 9, pp. 95-130.

[12] Maroni P., Fonctions Eulériennes, Polynômes Orthogonaux Classiques. Techniques de l'Ingénieur, Traité Généralités (Sciences Fondamentales)., 1994, no. A 154 Paris., pp. 1-30.

[13] Mason J.J., Chebychev polynomials of the second, third and fourth kinds in approximation, indefinite integration, and integral transforms. J. Comput. Appl. Math., 1993, no. 49, pp. 169-178.

DOI: https://doi.org/10.1016/0377-0427(39)90148-5.

[14] Mesquita T.A., Macedo A., Chebyshev polynomials via quadratic and cubic decompositions of the canonical sequence. Integral Transforms Spec. Funct., 2015, no. 26(12), pp. 956-970.

DOI: https://doi.org/10.1080/10652469.2015.1073274.

[15] Szegö G., Orthogonal Polynomials. Fourth edition, Amer. Math. Soc. Colloq. Publ., Vol. 23, Amer. Math. Soc., Providence, Rhode Island, 1975.

Received May 13, 2020.

In revised form, November 10, 2020.

Accepted November 16, 2020.

Published online December 16, 2020.

H. Merzouk

LMAM Laboratory and Department of Mathematics

Mohamed Seddik Ben Yahia University, Jijel, Algeria merzoukhind07@gmail.com

B. Aloui

Gabes University, Higher Institute of Industrial Systems of Gabes

Department of Electromechanics

Street Salah Eddine Elayoubi 6033 Gabes, Tunisia

Baghdadi.Aloui@fsg.rnu.tn

\section{A. Boussayoud}

Laboratory and Department of Mathematics,

Mohamed Seddik Ben Yahia University, Jijel, Algeria

alboussayoud@gmail.com 